\title{
Principal Message
}

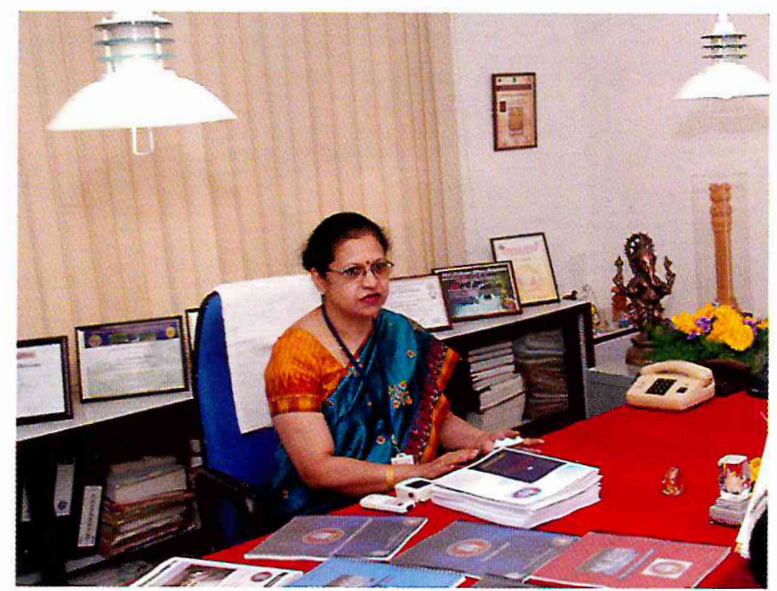

As the Principal of CODS, it gives me immense pleasure to announce the publish of yet another issue of CODS JOURNAL OF DENTISTRY. Our institute has come to be rightfully recognized in Karnataka and beyond, as a premier institute delivering excellence in education and patient care. Since its inception, we have tirelessly worked towards sustaining our benchmark as a leader and pioneer in education and today, we enjoy high repute and prestige. Our excellence in academics and related area has been recognized by NAAC by accreditation to our institute.

The collective endeavour of the students and our highly efficient team of staff members in bringing forth this journal with its immense wealth of knowledge adds another feather in the cap of the list of achievements of our college.

We pursue a culture of innovative learning through research and voluntary study. Our postgraduate programme serves as an opportunity for advanced research, with the easy availability of latest materials and state of the art analytical techniques at their easy dispense. Not only the post graduates but the undergraduate students have also been forthcoming in their contribution towards this journal, an attribute unheard of in other colleges and I highly appreciate the efforts of these students as well as the teachers in igniting the interests of these young minds.

In today's time when research is growing in leaps and bounds, it is imperative for us to be abreast with the latest innovations and thus in keeping with this view, we bring forth this edition of our journal. And I hope that it is well utilized by everyone. 